\title{
Biosensors and Bioelectronics Open Access
}

\section{An Electrochemical Aptasensor Based on Azophloxine-Graphene/ Gold Nanoparticles Composite for Sensitive Detection of ATP}

\author{
Yuxuan Wang ${ }^{1,2}$, Xuewen Ma ${ }^{1}$, Yanxiu Guo ${ }^{1}, \mathrm{Li}$ Wang $^{1 *}$ \\ ${ }^{1}$ Institute of Environmental Science, Shanxi University, China \\ ${ }^{2}$ Department of Applied Biology \& Chemical Technology, The Hong Kong Polytechnic University, Hong Kong
}

"Corresponding author: Li Wang, Assistant Professor, Institute of Environmental Science, Shanxi University, Taiyuan City, Shanxi Province, China. Tel: +863517011011; Email: wangli@sxu.edu.cn

Citation: Wang Y, Ma X, Guo Y, Wang L (2018) An Electrochemical Aptasensor Based on Azophloxine-Graphene/Gold Nanoparticles Composite for Sensitive Detection of ATP. Biosens Bioelectron Open Acc: BBOA-112. DOI: 10.29011/BBOA-112.100012

Received Date: 11 January, 2018; Accepted Date: 24 January, 2018; Published Date: 31 January, 2018

\begin{abstract}
This paper describes an alternative method for ATP detection. Azophloxine Functionalized Graphene/Gold Nanoparticles Composite (AP-Gr/AuNPs) was easily synthesized with the integrated properties of high conductivity, large specific surface area, electroactive function, and good biocompatibility from Azophloxine-Graphene/AuNPs. An electrochemical aptasensor for the specific and sensitive detection of Adenosine Triphosphate (ATP, as model analyte) was developed by using azophloxine as an in-situ electrochemical probe based on this nanocomposite. By coupling the high binding affinity and specificity of aptamer towards other molecules, the AP-Gr/AuNPs based aptasensor showed a great performance for the detection of ATP with a linear response from $1.0 \times 10^{-15}$ to $1.0 \times 10^{-12} \mathrm{M}$, also with a low detection limit of $3.3 \times 10^{-16} \mathrm{M}$. As compared with the routine luciferinluciferase method for ATP, this electrochemical aptasensor has a high sensitity and specifity towards ATP, which may has a good potential for the practical application of electrochemical aptasensors based on graphene and metal nanomaterials.
\end{abstract}

Keywords: Aptasensor; Biosensor; Graphere; Nanoparticle

\section{Introduction}

Adenosine Triphosphate (ATP) is a complex molecule containing the nucleoside adenosine and a tail consisting of three phosphates. In living cells, ATP is a critically important energy source that powers many intracellular reactions, which includes the synthesis of macromolecules, such as DNA and RNA, and proteins [1]. In addition to its central role in cellular metabolism, ATP is now recognized as an important extracellular signalling agent, which binds to a large family of membrane proteins [2-5]. Although the luciferin-luciferase method has long been known as a highly sensitive and selective method for measuring ATP, Luciferase enzymes are active in their native state and lose activity upon unfolding or denaturation, difficult to use in vivo [6]. Therefore, there is a need for the straightforward direct quantitative methods for ATP measurement.

An aptamer, a nucleic acid or peptide molecule, has been engineered through a selection process to exhibit exceptional binding affinity and specificity to their target or antigen. Because of the thermally stability, reusability and easy modification for their detection and immobilisation by introducing functional groups, aptamer-based sensors have been broadly used in detection of cancer cells, organic molecules, and a variety of proteins [7-11]. Among them, electrochemical aptamer-based sensors are widely employed due to their excellent sensitivity, rapid response, simplicity and low cost [12-14]. Electrochemical sensor for ATP as an alternative method could offer a wide range of applicability in physiological studies especially those performed in vivo. Some direct electro-chemical measurements of nucleotides have been reported with microelectrodes by sinusoidal voltammetry $[6,12]$. In order to enhance the sensitivity and specificity of the electrochemical sensor, some composite materials with high electrical conductivity coupled with aptamers with specific binding affinity towards ATP have to be employed to modify electrode.

In this paper, a different detection scheme is proposed based on electrochemistry with modified electrode by Azophloxine Functionalized Graphene/Gold Nanoparticles Composite (AP-Gr/AuNPs). By combining the great 


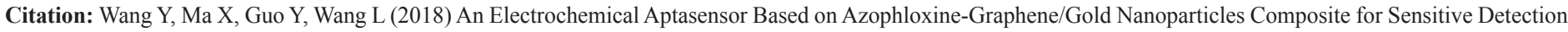
of ATP. Biosens Bioelectron Open Acc: BBOA-112. DOI: 10.29011/BBOA-112.100012

properties of the as obtained AP-Gr/AuNPs (high conductivity, high specific surface area, excellent biocompatibility and electroactive function) with aptamer (high affinity and specificity), a sensitive electrochemical aptasensing platform for the detection of Adenosine Triphosphate (ATP) has been developed.

\section{Experimental Section}

The general preparation and sensing procedure of this label-free electrochemical aptasensor are shown in Scheme 1. Firstly, AP-Gr was produced by the reduction of graphene oxide with hydrazine heated to about $60^{\circ} \mathrm{C}$. Then, Poly (Diallyldimethyl-Ammonium Chloride) (PDDA) and $\mathrm{HAuCl}_{4}$ aqueous solution were slowly added into the APGr solution under vigorous stirring at room temperature during 10 min. After addition, $\mathrm{NaBH}_{4}$ aqueous solution was introduced into above solution, and then the crude reaction mixture was left in water bath at $98^{\circ} \mathrm{C}$ for $20 \mathrm{~min}$ to achieve AP-Gr/AuNPs. The glassy carbon electrode was modified by dropping AP-Gr/AuNPs on the GCE surface to obtain APGr/AuNPs/GCE. Then, the thiolated ATP-Binding Aptamer (HS-ABA) was immobilized on the surface of AP-Gr/AuNPs/ GCE by the formation of Au-S bond to achieve the sensing interface of HS-ABA/AP-Gr/AuNPs/GCE. The Differential Pulse Voltammetry (DPV) signal of AP decreased because the electron transfer was retarded by nucleobases. After the immolation of thiolated ATP-binding aptamer, the sensing interface was immersed into ATP solution for $20 \mathrm{~min}$. Finally, the label-free electrochemical aptasensor for ATP detection was developed. The DPV signals decreased due to the formation of ABA-ATP complex on the surface of electrode. The detection linear range is from $1.0 \times 10^{-15}$ to $1.0 \times 10^{-12} \mathrm{M}$, and the detection limit is as low as $3.3 \times 10^{-16} \mathrm{M}$. The detection sensitivity was enhanced by redox electrochemical material of AP-Gr/AuNPs. There is no need for the sophisticated and expensive procedures for the labelling of aptamers.

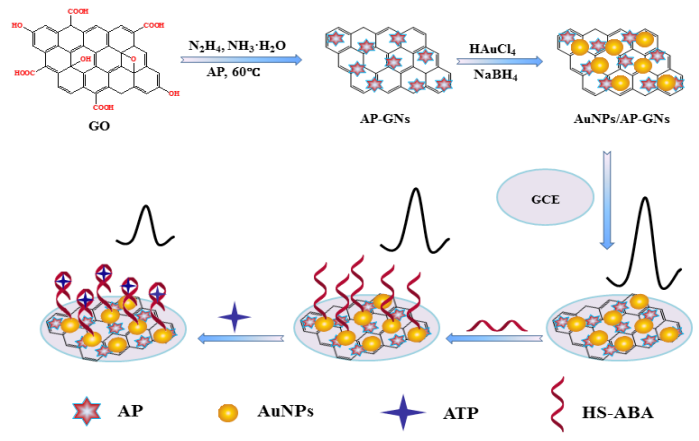

Scheme 1: Schematic illustration of the fabrication of AP-Gr/AuNPs and the electrochemical sensing procedure of ATP.

\section{Materials and Reagents}

DNA oligonucleotides, Adenosine Triphosphate (ATP), Cytidine Triphosphate (CTP), Guanosine Triphosphate (GTP), Uridine Triphosphate (UTP) and the oligonucleotide sequences include 5'-thiolated ATP-binding aptamer (HSABA): 5'-SH- $\left(\mathrm{CH}_{2}\right)_{6}$-ACC TGG GGG AGT ATT GCG GAG GAA GGT-3', Mutant ATP Binding Aptamer (MABA): 5'SH- $\left(\mathrm{CH}_{2}\right)_{6}$-ATA TCA CCT GGG GGA AGG TCT GTA-3' were purchased from Sangon Inc. (Shanghai, China). Graphite was purchased from Alfa Aesar. 6-Mercapto-L-Hexanol $(\mathrm{MCH})$, Tris (2-Carboxyethyl) Phosphine Hydrochloride (TCEP), PDDA (Mw $=400000-500000,20 \mathrm{wt} \%$ in water) were obtained from Sigma Co. Azophloxine was purchased from Beijing Xuanwu Chemical Factory. Hydrazine solution (50 wt $\%$ ), ammonia solution (25-28 wt\%), $\mathrm{HAuCl}_{4}$ were obtained from Beijing chemical reagent factory (Beijing, China). HSABA and MABA were dissolved in Tris- $\mathrm{HCl}$ buffer $(20 \mathrm{mM}$ Tris- $\mathrm{HCl}, 0.10 \mathrm{M} \mathrm{NaCl}, 5.0 \mathrm{mM} \mathrm{MgCl} \cdot 6 \mathrm{H}_{2} \mathrm{O}, 40 \mu \mathrm{M}$ TCEP, $\mathrm{pH}$ 7.4). Tris buffer solution ( $20 \mathrm{mM}$ Tris- $\mathrm{HCl}, 0.10 \mathrm{M} \mathrm{NaCl}$ and $\left.5.0 \mathrm{mM} \mathrm{MgCl} \cdot 6 \mathrm{H}_{2} \mathrm{O}, \mathrm{pH} 7.4\right)$ was used as electrolyte for electrochemical measurement of ATP. Fresh human whole blood was obtained from the local hospital. Water for all experiments was purified with the Millipore system.

\section{Instruments}

Transmission Electron Microscopy (TEM) images were obtained with a JEM-1011 transmission electron microscope operating at $100 \mathrm{kV}$. X-Ray Photoelectron Spectroscopy (XPS) measurement was performed on an ESCALABMKII 250 photoelectron spectrometer (VG Co.) with Al $\mathrm{K} \alpha \mathrm{X}$-ray radiation as the $\mathrm{X}$-ray excitation source. UV-Vis absorption spectra were recorded on a U-3010 spectrometer (Hitachi, ltd. Tokyo Japan). Electrochemical measurements were conducted on CHI660C electrochemical workstation (Chenhua Instruments Co., Shanghai, China). A threeelectrode system was used for the experiment with the Glassy Carbon Electrode (3 $\mathrm{mm}$ in diameter) (GCE) as the working electrode, a Saturated Calomel Electrode (SCE) and a Pt wire electrode were used as reference and counter electrode, respectively.

\section{Synthesis of AP-Gr}

Graphene Oxide (GO) was synthesized from natural graphite by Hummers' method with some modification [15]. The AP-Gr was synthesized with reference to the reported procedure from literature [16]. Briefly, $7.4 \mathrm{~mL}$ of $0.50 \mathrm{mg} /$ $\mathrm{mL}$ aqueous azophloxine solution was mixed with $2.5 \mathrm{~mL}$ of $1.0 \mathrm{mg} / \mathrm{mL}$ GO in $15 \mathrm{~mL}$ flask, and then $100 \mu \mathrm{L}$ of ammonia solution and $10 \mu \mathrm{L}$ of hydrazine solution were added into the flask. After well-mixed by vigorously stirring for a few 


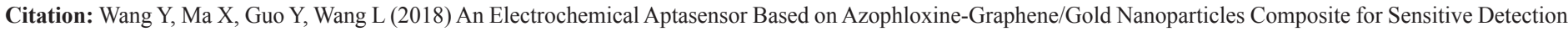
of ATP. Biosens Bioelectron Open Acc: BBOA-112. DOI: 10.29011/BBOA-112.100012

minutes, the reaction mixture was heated in a water bath $\left(60^{\circ} \mathrm{C}\right)$ for 4 hours. After the stable black disperse system was formed, AP-Gr was collected by the filtration of disperse system through a Nylon membrane $(0.22 \mu \mathrm{m})$, which can be re-dispersed readily in water by ultrasonication. Pure graphene was prepared with the same procedure as that of AP-Gr without the addition of azophloxine.

\section{Preparation of AP-Gr/AuNPs}

Firstly, $125 \mu \mathrm{L}$ PDDA (20 wt\%) was added into $4.0 \mathrm{~mL}$ of $0.12 \mathrm{mg} / \mathrm{mL}$ AP-Gr. Then, $250 \mu \mathrm{L} 0.010 \mathrm{~g} / \mathrm{mL} \mathrm{HAuCl}_{4}$ was added into the above solution and stirred at room temperature for $10 \mathrm{~min}$. Finally, $200 \mathrm{uL} 10 \mathrm{mM} \mathrm{NaBH}_{4}$ was added into the above solution and the reaction mixture was heated in a water bath at $98^{\circ} \mathrm{C}$ for $20 \mathrm{~min}$. The AP-Gr/AuNPs generated in the reaction mixture was collected by centrifugation.

\section{Fabrication of the Sensing Interface}

GCE was polished to a mirror-like surface with 1, 0.3, and $0.05 \mu \mathrm{m}$ alumina powder sequentially and then rinsed thoroughly with redistilled water between each polishing step, then washed sequentially with $1: 1$ nitric acid, acetone and redistilled water in ultrasonic bath, and then left it to dry in air. AP-Gr/AuNPs modified GCE (AP-Gr/AuNPs/GCE) was obtained by dropping $6.0 \mu \mathrm{L}$ AP-Gr/AuNPs on the surface of GCE and keeping it to dry at room temperature. The sensing interface of HS-ABA/AP-Gr/AuNPs/GCE was obtained by dropping $7.0 \mu \mathrm{L}$ of $5.0 \mu \mathrm{M}$ HS-ABA on the surface of AP-Gr/ AuNPs/GCE, and then kept it to dry at $4^{\circ} \mathrm{C}$ overnight.

\section{Electrochemical Detection of ATP}

As well known, ATP is an extracellular signalling mediator that occur in all living cells for biological processes and is essential for biomass determinations in diagnosis and therapy of associated disease, food quality control and environmental analyses [17]. Therefore, there is a great need to develop a fast and less sophisticated procedure for ATP detection. Differential Pulse Voltammetry (DPV) was employed to record the electrochemical signal of AP in tris buffer solution. Firstly, HS-ABA/AP-Gr/ AuNPs/GCE was immersed into $1.0 \mathrm{~mL} 100 \mu \mathrm{M} \mathrm{MCH}$ for 2 hours to block the possible remaining active sites and then the electrode was rinsed with tris buffer. Then, the as-prepared sensing interfaces was put into ATP solution at different concentrations for $40 \mathrm{~min}$. and the decreased signal of AP was measured by DPV in Tris buffer solution. For interference experiment, ATP solution was replaced with $1.0 \times 10^{-13} \mathrm{M}$ GTP, UTP, CTP solution, respectively, with the other procedures being the same as that for ATP detection. Another control experiment was also conducted by using HS-MABA, which severely reduced the binding activity in the presence of ATP by a changed aptamer sequence, instead of HS-ABA to modify the AP-Gr/AuNPs/GCE, followed with incubation in $1.0 \times 10^{-13} \mathrm{M}$ ATP for $40 \mathrm{~min}$. After that, the DPV signal of AP was measured.

\section{Application of the ATP Aptasensor in Serum Samples}

The applicability and reliability of this electrochemical aptasensor was evaluated by the detection of ATP in real human serum. Fresh human whole blood was obtained from the local hospital and the sample was diluted by 100 times with tris buffer solution. The experimental procedures were the same as previously reported for the ATP detection [18].

\section{Results and Discussions}

\section{Characterization of AP-Gr/AuNPs}

The absorption spectra of AP, GO, AP-Gr and AP-Gr/AuNPs along with the calculated spectra of AP-Gr (f) and AP-Gr/AuNPs (h) were shown in Figure 1. The spectrum of AP (red) exhibits strong absorptions at 504 and $531 \mathrm{~nm}$ [19]. The GO suspension has a characteristic absorption band at $228 \mathrm{~nm}$ (grey), arising from the $\pi-\pi *$ transition of aromatic $\mathrm{C}=\mathrm{C}$ bonds, and a shoulder at $\sim 290-300$ $\mathrm{nm}$ assigned to the $\mathrm{n}-\pi^{*}$ transition of the $\mathrm{C}=\mathrm{O}$ bond $[20,21]$. The absorption bands of AP-Gr (orange) display a significant broadening along with red-shift as compared with the spectral addition of AP and GO. The red-shift of absorption band to $263 \mathrm{~nm}$ is resulted from the reduction of graphene oxide. The other low intensity absorption band at about $553 \mathrm{~nm}$ has a significant red shift of 30 $\mathrm{nm}$ as compared with the absorption of AP. The strong electronic interaction between AP and GO molecules suggests that AP were integrated with graphene nanosheets by inter-molecular forces and $\pi-\pi$ interaction between GO and AP [22]. Since AP was introduced before GO was reduced to overcome the aggregation, AP would be expected to adsorb on the surface of GO via inter-molecular forces and interaction. After GO reduction, the $n-\pi^{*}$ transition is almost disappeared in the absorption spectrum of AP-Gr, which suggests that GO was reduced. After the incorporation of AuNPs into APGr, there are two obvious absorption bands centred at 261 and 523 $\mathrm{nm}$ (green), which were consistent with the absorption of graphene and AuNPs [23,24], respectively. The comparison of absorption spectra suggests that AuNPs could be successfully integrated into the surface of AP-Gr. The confirmation awaits the further evidence which will be discussed in the following. 
Citation: Wang Y, Ma X, Guo Y, Wang L (2018) An Electrochemical Aptasensor Based on Azophloxine-Graphene/Gold Nanoparticles Composite for Sensitive Detection of ATP. Biosens Bioelectron Open Acc: BBOA-112. DOI: 10.29011/BBOA-112.100012

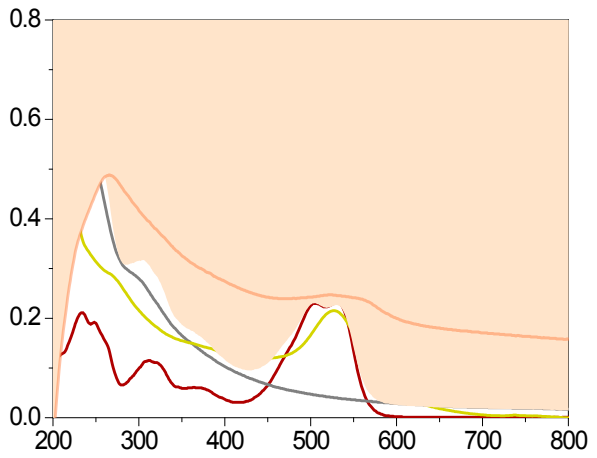

Figure 1: UV-Vis spectra of azophloxine solution (red), GO suspension (grey), Au nanoparticles (gold), AP-Gr suspension (orange) and AP-Gr/AuNPs suspension (green), along with the calculated spectra of AP-Gr (f), and AP-Gr/AuNPs (h) by the same method as that from reference [25].

Figure 2A shows the TEM images of AP-Gr and AP-Gr/ AuNPs. The TEM image of AP-Gr has the flake-like shape of graphene with some wrinkled distortions resulted from the extremely thin layer AP-Gr nanosheets. The TEM image of AP-Gr/AuNPs nanocomposites as shown in Figure 2B, AuNPs were uniformly distributed on the network of AP-Gr.

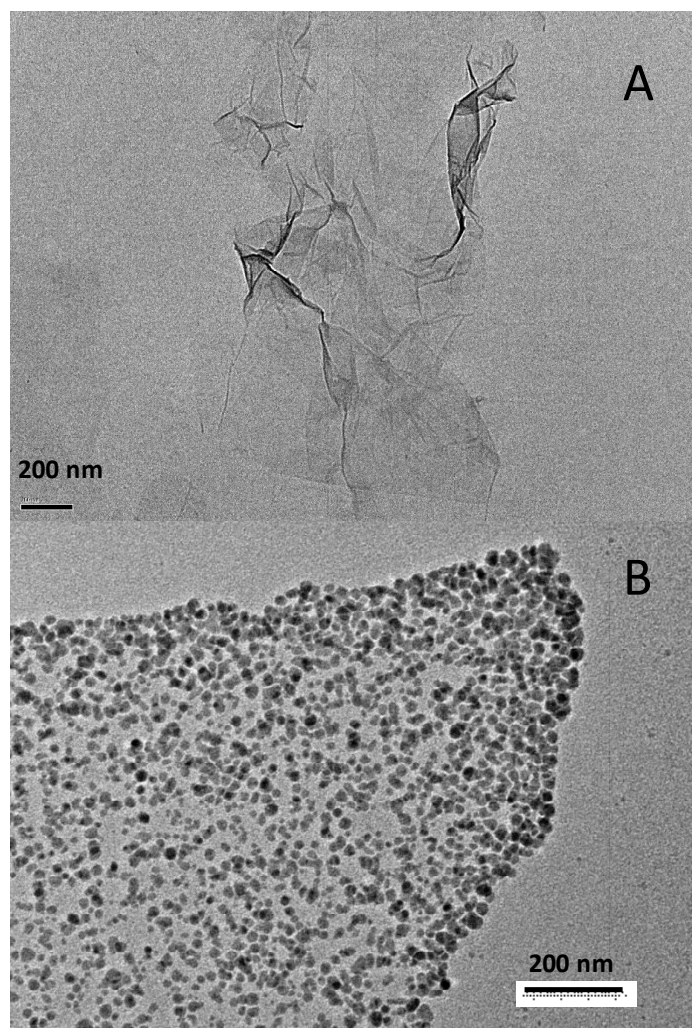

Figures 2A-B: The TEM image of AP-Gr (A) and AP-Gr/AuNPs (B).
The surface chemical composition and chemical state of AuNPs/O-GNs were further analysed by XPS. The XPS spectrum of AP-Gr/AuNPs with peak fitting using the Shirley algorithm to construct the background are shown in Figure 3A. The peak of Au4f originates from AuNPs, and the peak of $\mathrm{C} 1 \mathrm{~s}$ arises from graphene nanosheets and PDDA. The peak of N1s is from AP and PDDA, and S2p from AP. As shown in Figure $3 \mathrm{~B}$, the doublet of $\mathrm{Au} 4 \mathrm{f}_{5 / 2}$ and $\mathrm{Au} 4 \mathrm{f}_{7 / 2}$ having binding energies at 87.1 and $83.5 \mathrm{eV}$ with peak-to-peak separation of $3.6 \mathrm{eV}$ verifies that $\mathrm{Au}$ nanoparticles have been formed by the reduction of $\mathrm{NaBH}_{4}$ [26]. The C1s XPS spectrum of AP$\mathrm{Gr} / \mathrm{AuNPs}$ is shown in Figure 3C. It displays the presence of four main types of carbon bonds: C-C (284.6 eV), C-N (285.7 $\mathrm{eV}), \mathrm{C}-\mathrm{O}(286.4 \mathrm{eV})$ and C-S $(287.4 \mathrm{eV})$. The peak intensity of oxygen-containing functional groups, such as $\mathrm{C}=\mathrm{O}(287.6$ $\mathrm{eV})$, become very low, barely discernible. As compared with the C1s XPS spectra of GO $[27,28]$, it indicates the effective reduction of $\mathrm{GO}$ to graphene.
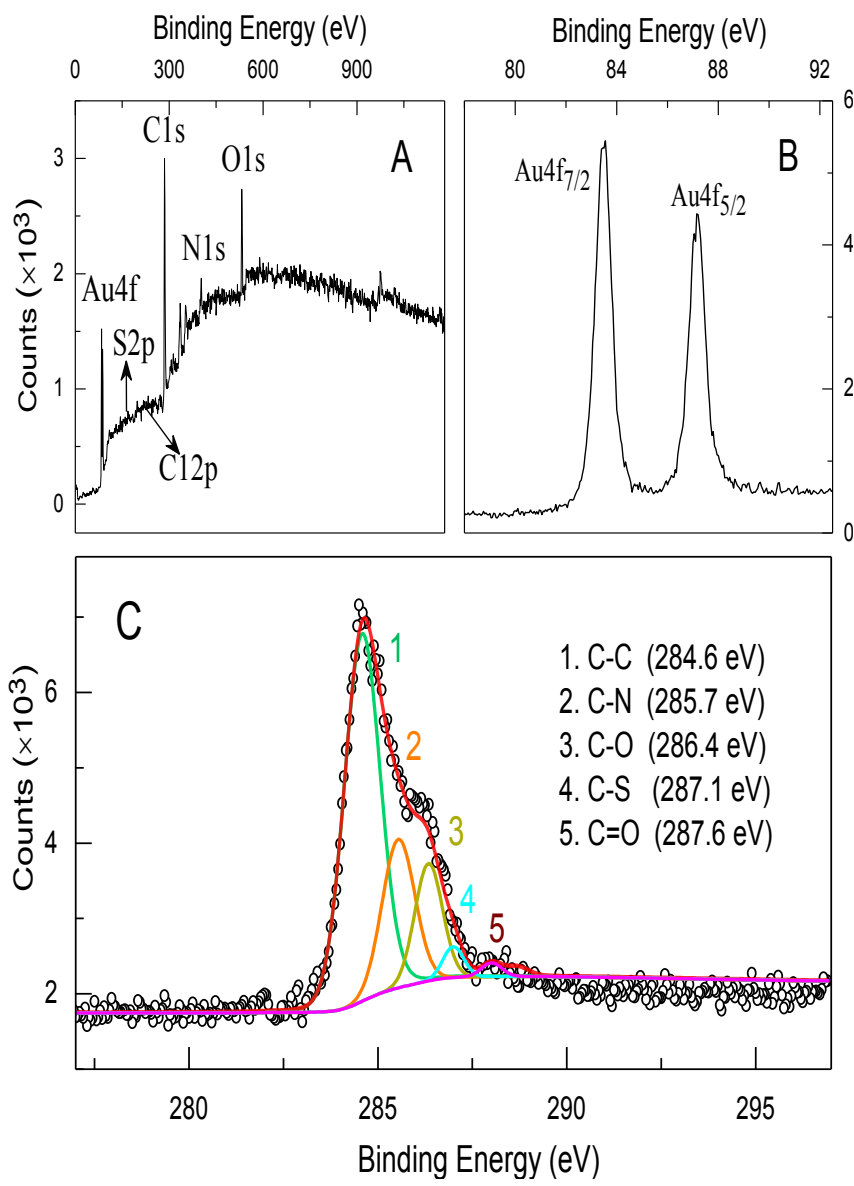

Figures 3A-C: The survey XPS data for AP-Gr/AuNPs (A) and $\mathrm{Au}(4 \mathrm{f})(\mathrm{B}),(\mathrm{C})$ is C1s XPS spectra of AP-Gr/AuNPs, (D) The Raman spectra of AP-Gr (a), GO (b), AP-Gr/AuNPs(c) and AP(d). 


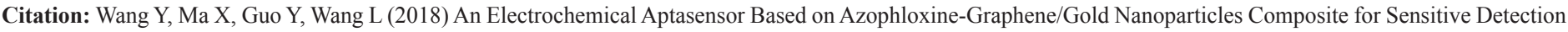
of ATP. Biosens Bioelectron Open Acc: BBOA-112. DOI: 10.29011/BBOA-112.100012

The sensing interface from the modified electrode surface was characterized by cyclic voltammetry. As shown in Figure 4A, there was no redox peaks from the Cyclic Voltammograms (CV) of GCE (curve a) in tris buffer solution. When GCE was modified with AP-Gr/GCE, a pair of well-defined redox peaks clearly appeared in the investigated potential range (curve b). The redox peaks arose from AP. As compared with the CV of AP-Gr/GCE, the peak currents dramatically increased with the AP-Gr/AuNPs/GCE (curve c). With reference to the reported CV data by Guo et al. [22] the peak currents of AP-Gr/AuNPs/ GCE was relatively higher than that of AP-Gr/GCE. The efficient interfacial electron-transfer process maybe facilitated by the presence of AuNPs through a catalytic effect.

\section{Electrochemical Detection of ATP}

The electrochemical property of AP-Gr/AuNPs modified electrode was further investigated by DPV technique. As expected, AP-Gr/AuNPs modified GCE demonstrated significant DPV response as shown in Figure 4B. When further modified with HS-ABA, the DPV signal of HS-ABA/AP-Gr/AuNPs/GCE decreased remarkably (Figure 4B curve b). With reference to the binding curves reflecting the change of electron transfer resistance versus the aptamer concentrations [29], the decreased DPV signal with the further modification of HS-ABA suggested that HS-ABA has been successfully assembled on the sensing surface of AP-Gr/AuNPs/GCE. The DPV signal further decreased (Figure 4B curve c) when the HS-ABA/AP-Gr/AuNPs/ GCE was immersed into MCH solution for 2 hours. This demonstrated that $\mathrm{MCH}$ further blocked the remaining active sites on the sensing interface, resulted in an increased electron transfer resistance. After that, the above-prepared aptasensor was placed into $5.0 \times 10^{-16} \mathrm{M}$ ATP solution and incubated for $40 \mathrm{~min}$.

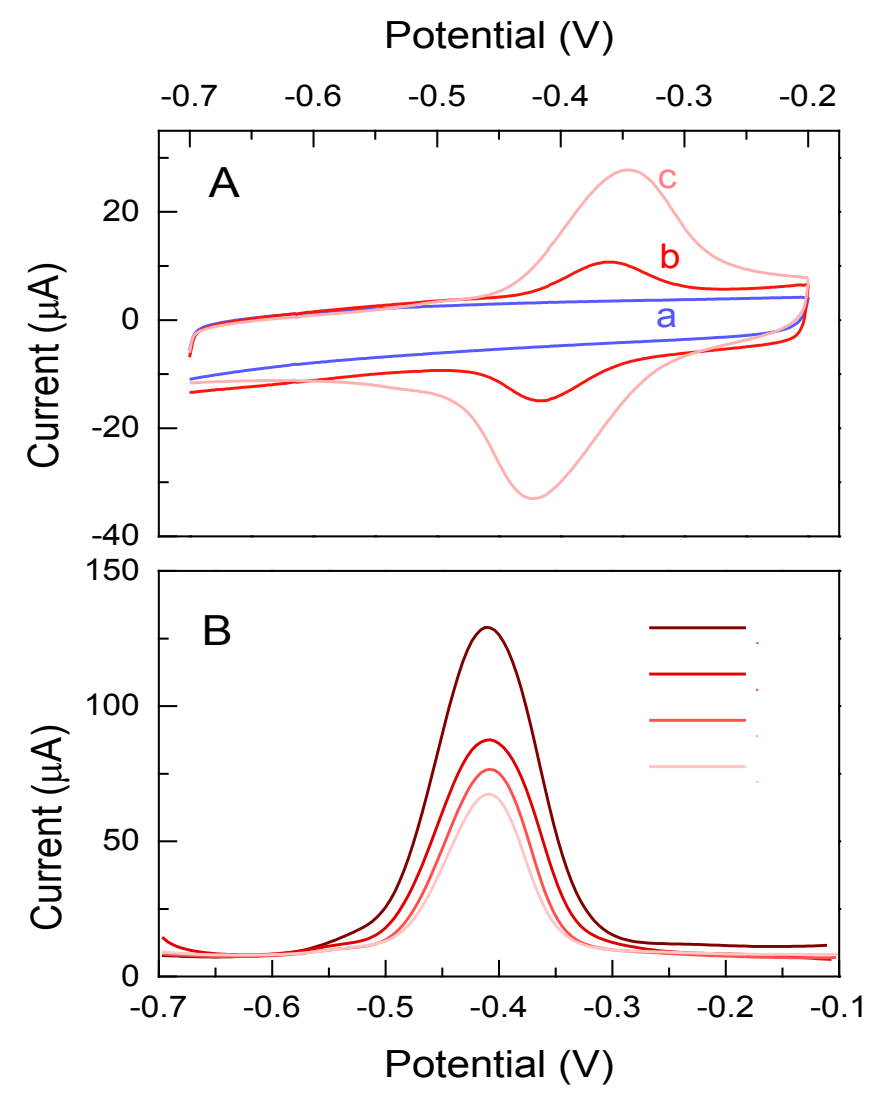

Figures 4A-B: (A) The CVs of GCE (a), AP-Gr/GCE (b), AP-Gr/AuNPs/GCE (c) in Tris buffer at a scan rate of $0.05 \mathrm{~V} \mathrm{~s} \mathrm{~s}^{-1}$. (B) The DPV characterization of AP-Gr/AuNPs/GCE (a), HS-ABA/AP-Gr/AuNPs/GCE (b), and MCH/HS-ABA/AP-Gr/AuNPs/GCE (c), after adding ATP (d) in Tris buffer at a scan rate of $0.05 \mathrm{~V} \mathrm{~s}^{-1}$. 
Citation: Wang Y, Ma X, Guo Y, Wang L (2018) An Electrochemical Aptasensor Based on Azophloxine-Graphene/Gold Nanoparticles Composite for Sensitive Detection of ATP. Biosens Bioelectron Open Acc: BBOA-112. DOI: 10.29011/BBOA-112.100012

The peak current decreased further ((Figure 4B) curve d), suggesting that the formed ABA-ATP complex as another insulating layer added more resistance for the interfacial charge transfer. The peak current of DPV signals was linearly correlated with the logarithmic concentration of ATP solution (Figure 5). The linear range was from $1.0 \times 10^{-15}$ to $1.0 \times 10^{-12} \mathrm{M}$ with a correlation coefficient of 0.99 .

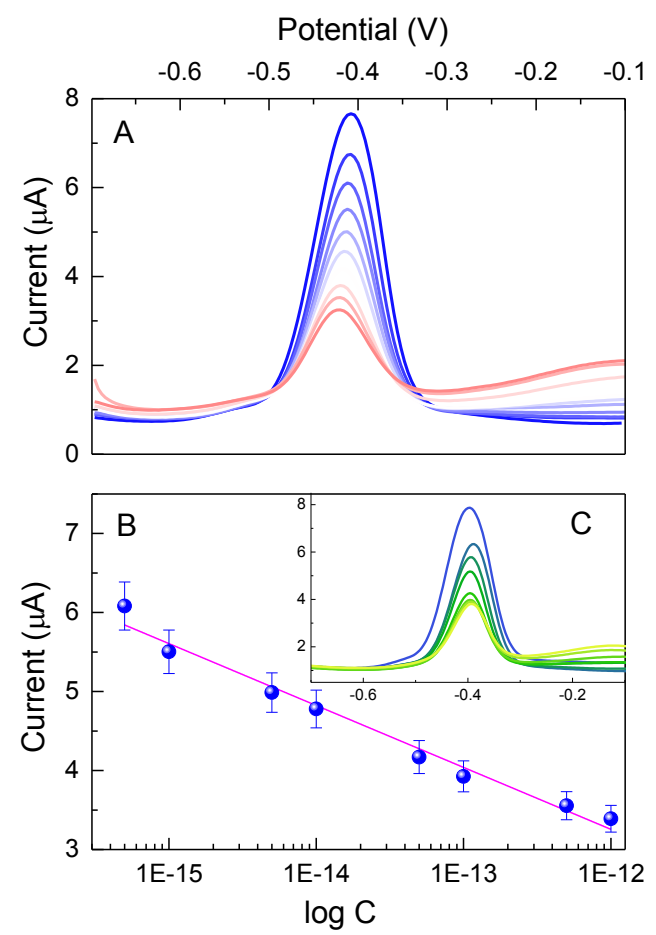

Figure 5: (A) DPV of MCH/HS-ABA/AP-Gr/GCE (a) and the relationship between current response and ATP concentration (from b to $\mathrm{j}$ : $1.0 \times 10^{-16}, 5.0 \times 10^{-16}, 1.0 \times 10^{-15}, 5.0 \times 10^{-15}, 1.0 \times 10^{-14}, 5.0 \times 10^{-14}$, $\left.1.0 \times 10^{-13}, 5.0 \times 10^{-13}, 1.0 \times 10^{-12} \mathrm{M}\right)$. (B) The calibration curve between the current response and ATP concentration (logarithm) in tris buffer. Inset is the DPVs acquired in the real samples in the presence of ATP.

The standard deviation was less than $5 \%$, calculated from three different measurements. The detection limit was $3.3 \times 10^{-16} \mathrm{M}$ given the signal-to-noise ratio $\mathrm{S} / \mathrm{N}=3$, that is more sensitive or comparable with the previous electrochemical aptasensors for ATP detection [30-33], as listed in Table 1. The outstanding properties of HS-ABA/AP-Gr/AuNPs/ GCE sensor is attributed to the excellent electronic transport property along with the large specific surface area of graphene and AuNPs, as well as the high affinity and specificity of aptamer towards ATP.

\begin{tabular}{|cc|c|c|}
\hline Detection method & Linear range & $\begin{array}{c}\text { Detection } \\
\text { limit }\end{array}$ & $\begin{array}{c}\text { Refer } \\
\text { ences }\end{array}$ \\
\hline AuNPs/rGO-NH2/GCE & $10 \mathrm{pM}-100 \mathrm{nM}$ & $10 \mathrm{pM}$ & {$[7]$} \\
\hline Ru-SiNPs/Au electrode & $1.0 \mathrm{pM}-1.0 \mathrm{nM}$ & $0.2 \mathrm{pM}$ & {$[31]$} \\
\hline tris(bpyRu)- $\beta-\mathrm{CD} / \mathrm{GCE}$ & $50 \mathrm{pM}-10 \mathrm{nM}$ & $10 \mathrm{pM}$ & {$[32]$} \\
\hline $\begin{array}{c}\text { CdS-DNA concatamer- } \\
\text { based aptasensor }\end{array}$ & $0.1 \mathrm{pM}-10 \mathrm{nM}$ & $0.06 \mathrm{pM}$ & {$[33]$} \\
\hline AP-Gr/AuNPs/GCE & $1.0 \mathrm{fM}-1.0 \mathrm{pM}$ & $0.33 \mathrm{fM}$ & this work \\
\hline
\end{tabular}

Table 1: Comparison of the analytical parameters with other electrochemical aptasensors for ATP detection.

\section{Selectivity and Stability of Aptasensor}

Toverify the selectivity of this aptasensor, some analogues of ATP, such as Cytidine Triphosphate (CTP), Guanosine Triphosphate (GTP), and Uridine Triphosphate (UTP) were used in the interference experiment as control. The sensing electrode were immersed in $1.0 \times 10^{-13} \mathrm{MCTP}, 1.0 \times 10^{-13} \mathrm{MGTP}$, $1.0 \times 10^{-13}$ M UTP and $1.0 \times 10^{-14}$ M ATP for $40 \mathrm{~min}$, respectively, and then the DPV signals were recorded. As shown in Figure 6 , only ATP can cause a significant current decrease. This demonstrated that the formation of ATP-HS-TBA complex caused ATP molecules attached on the sensing interface.

MABA (which had a change in sequence that severely reduced the binding activity in the presence of thrombin) was also used as another control experiment to assess the selectivity of this aptasensor. The assembly processing was the same other than using MABA instead of HS-ABA to form sensing interface. Then the sensing interface was incubated into $1.0 \times 10^{-14}$ ATP for $40 \mathrm{~min}$. As shown in Figure 6, MABA did not show obvious current decrease in the presence of ATP. This confirmed that ATP could not interact with MABA effectively. The results of these experiments demonstrated the good selectivity of this aptasensor.

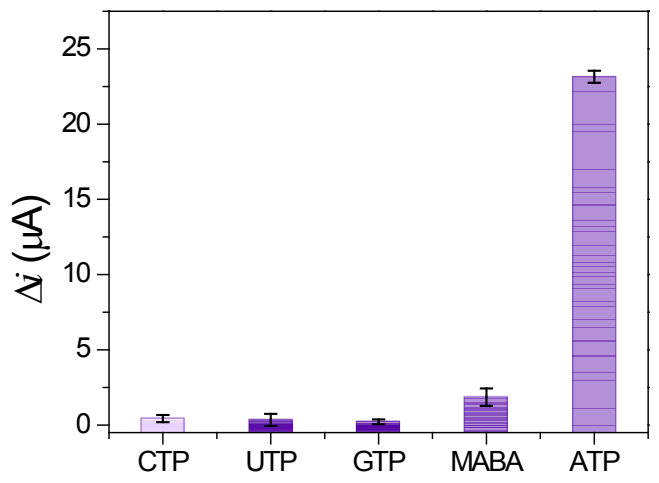

Figure 6: The DPV signal response upon addition of $1.0 \times 10^{-13} \mathrm{M}$ CTP, $1.0 \times 10^{-13}$ M GTP, $1.0 \times 10^{-13}$ M UTP and $1.0 \times 10^{-14} \mathrm{M}$ ATP. The 
Citation: Wang Y, Ma X, Guo Y, Wang L (2018) An Electrochemical Aptasensor Based on Azophloxine-Graphene/Gold Nanoparticles Composite for Sensitive Detection of ATP. Biosens Bioelectron Open Acc: BBOA-112. DOI: 10.29011/BBOA-112.100012

error bars represent the standard deviation of three measurements.

To investigate the stability of the aptasensor, the modified electrode with sensing interface was stored in ultrapure water at $4^{\circ} \mathrm{C}$ over 2 weeks and then brought back to room temperature slowly. The DPV signal of the stored electrode with modified sensing interface changed slightly as compared with the signal before the storage over 2 weeks. Apparently, this electrochemical aptasensor possesses good stability for ATP detection.

\section{Recovery Test}

The recovery test was conducted to investigate the possible application of this new electrochemical aptasensor in clinical analysis for complex systems. Human serum samples were used as a model complex system. The blood sample was diluted one hundred times by tris buffer solution. Table 2 showed the results of three samples. The favourable recovery data clearly indicated this aptasensor could be used for the detection ATP in practical samples with great selectivity and sensitively.

\begin{tabular}{|c|c|c|c|c|}
\hline Samples & $\begin{array}{c}\text { Added } \\
(\mathbf{p M})\end{array}$ & $\begin{array}{c}\text { Found } \\
(\mathbf{p M})\end{array}$ & Recovery\% & RSD\% \\
\hline $\mathbf{1}$ & 0.01 & 0.0101 & 101.0 & 2.8 \\
\hline $\mathbf{2}$ & 0.10 & 0.0999 & 99.9 & 3.1 \\
\hline $\mathbf{3}$ & 1.00 & 0.9995 & 99.9 & 2.6 \\
\hline
\end{tabular}

Table 2: The recoveries of ATP from human serum samples.

\section{Conclusions}

This work has demonstrated a new electrochemical aptasensor for the detection of ATP by coupling the electrochemical properties of graphene, and aptamer conjugated AuNPs, as well as the high binding affinity and specificity of aptamer towards other molecules. The aptasensor fabricated without labelling at low cost. It has shown very sensitive responses to ATP with the detection limits of $3.3 \times 10^{-16} \mathrm{M}$, which were more sensitive than previously reported methods. The simplified strategy can be exploited to synthesize other electroactive metal-graphene materials and immobilize various aptamers for the detection of the corresponding targets. In conclusion, this work paves a promising way for the fabrication of functionalized graphene nanomaterial-based electrochemical aptasensor and may have good potentials for future practical applications.

\section{Acknowledgements}

This research work was supported by the Provincial Government for the Oversea Hundreds of talents Program
(No. 205107001-2015), the Program of 131 Leading Principal Investigator, (205544901006-2015), and the Returned Overseas Scholar Foundation of Shanxi Province (205586801004-2015).

\section{References}

1. Yaginuma H, Kawai S, Tabata KV, Tomiyama K, Kakizuka A, et al. (2014) Diversity in ATP concentrations in a single bacterial cell population revealed by quantitative single-cell imaging. Scientific Reports 4: 6522.

2. Jeter CR, Tang W, Henaff E, Butterfield T, Roux SJ (2004) Evidence of a Novel Cell Signaling Role for Extracellular Adenosine Triphosphates and Diphosphates in Arabidopsis. The Plant Cell 16: 2652-2664.

3. El-Moatassim C, Dornand J, Mani JC (1992) Extracellular ATP and cell signalling. Biochimica et Biophysica Acta (BBA) - Molecular Cell Research 1134: 31-45.

4. Fields RD, Stevens B (2000) ATP: an extracellular signaling molecule between neurons and glia. Trends Neurosci 23: 625-633.

5. Falzoni S, Donvito G, Di Virgilio F (2013) Detecting adenosine triphosphate in the pericellular space. Interface Focus 3: 20120101.

6. Llaudet E, Hatz S, Droniou M, Dale N (2005) Microelectrode Biosensor for Real-Time Measurement of ATP in Biological Tissue. Anal Chem 77: 3267-3273.

7. Zhu L, Liu Y, Yang P, Liu B (2015) Label-free Aptasensor based on Electrodeposition of Gold Nanoparticles on Graphene and Its Application in the Quantification of Adenosine Triphosphate. Electrochimica Acta 172: 88-93.

8. Zhou Q, Rahimian A, Son K, Shin DS, Patel T, et al. (2016) Development of an aptasensor for electrochemical detection of exosomes. Methods 97: 88-93.

9. $\quad$ Qi Y, Li B (2011) A Sensitive, Label-Free, Aptamer-Based Biosensor Using a Gold Nanoparticle-Initiated Chemiluminescence System. Chem Eur J 17: 1642-1648.

10. Rivas L, Mayorga-Martinez CC, Quesada-González D, ZamoraGálvez A, de la Escosura-Muñiz A, et al. (2015) Label-Free Impedimetric Aptasensor for Ochratoxin-A Detection Using Iridium Oxide Nanoparticles. Anal Chem 87: 5167-5172.

11. Cho EJ, Lee JW, Ellington AD (2009) Applications of Aptamers as Sensors. Annu Rev Anal Chem 2: 241-264.

12. Zhou L, Wang J, Li D, Li Y (2014) An electrochemical aptasensor based on gold nanoparticles dotted graphene modified glassy carbon electrode for label-free detection of bisphenol $A$ in milk samples. Food Chemistry 162: 34-40.

13. Jo H, Gu H, Jeon W, Youn H, Her J, et al. (2015) Electrochemical Aptasensor of Cardiac Troponin I for the Early Diagnosis of Acute Myocardial Infarction. Anal Chem 87: 9869-9875.

14. Zhengbo C, Junxia G, Jing L, Lin G, (2013) An electrochemical aptasensor based on the amplification of two kinds of gold nanocrystals for the assay of L-histidine with picomolar detection limit. Nanotechnology 24: 295501.

15. Hummers JWS, Offema RE (1958) Preparation of Graphitic Oxide. Journal of American Chemical Society 80: 1339-1339. 
Citation: Wang Y, Ma X, Guo Y, Wang L (2018) An Electrochemical Aptasensor Based on Azophloxine-Graphene/Gold Nanoparticles Composite for Sensitive Detection of ATP. Biosens Bioelectron Open Acc: BBOA-112. DOI: 10.29011/BBOA-112.100012

16. Guo YJ, Han YJ, Guo YX, Dong C (2013) Graphene-Orange II composite nanosheets with electroactive functions as label-free aptasensing platform for "signal-on" detection of protein. Biosensors \& Bioelectronics 45: 95-101.

17. Yu F, Li L, Chen F (2008) Determination of adenosine disodium triphosphate using prulifloxacin-terbium(III) as a fluorescence probe by spectrofluorimetry. Analytica Chimica Acta. 610: 257-262.

18. Chen X, Ge L, Guo B, Yan M, Hao N, et al. (2014) Homogeneously ultrasensitive electrochemical detection of adenosine triphosphate based on multiple signal amplification strategy. Biosensors and Bioelectronics 58: 48-56.

19. Foldvary CM, Wojnarovits $L$ (2007) The effect of high-energy radiation on aqueous solution of Acid Red 1 textile dye. Radiation Physics and Chemistry 76: 1485-1488.

20. Lai Q, Zhu S, Luo X, Zou M, Huang S (2012) Ultraviolet-visible spectroscopy of graphene oxides. AIP Advances 2: 032146.

21. Moon IK, Lee J, Ruoff RS, Lee H (2010) Reduced graphene oxide by chemical graphitization. Nature Communications 1: 6 .

22. Guo Y, Guo Y, Dong C (2013) Ultrasensitive and label-free electrochemical DNA biosensor based on water-soluble electroactive dye azophloxine-functionalized graphene nanosheets. Electrochimica Acta 113: 69-76.

23. Chen HJ, Wang YL, Wang YZ, Dong SJ, Wang EK (2006) Onestep preparation and characterization of PDDA-protected gold nanoparticles. Polymer 47: 763-766.

24. Lin XQ, Kang GF, Lu LP (2007) DNA/Poly(p-aminobenzensulfonic acid) composite bi-layer modified glassy carbon electrode for determination of dopamine and uric acid under coexistence of ascorbic acid. Bioelectrochemistry 70: 235-244.
25. Wang L, Ashford DL, Thompson DW, Meyer TJ, Papanikolas JM (2013) Watching Photoactivation in a Ru(II) Chromophore-Catalyst Assembly on TiO2 by Ultrafast Spectroscopy. The Journal of Physical Chemistry C 117: 24250-24258.

26. Jaramillo TF, Baeck SH, Cuenya BR, McFarland EW (2003) Catalytic activity of supported au nanoparticles deposited from block copolymer micelles. Journal of the American Chemical Society 125: 7148-7149.

27. Bai H, Xu Y, Zhao L, Li C, Shi G (2009) Non-covalent functionalization of graphene sheets by sulfonated polyaniline. Chem Commun: 1667-1669.

28. Xu Y, Bai H, Lu G, Li C, Shi G (2008) Flexible Graphene Films via the Filtration of Water-Soluble Noncovalent Functionalized Graphene Sheets. J Am Chem Soc 130: 5856-5857.

29. Eissa S, Zourob M (2017) Aptamer- Based Label-Free Electrochemical Biosensor Array for the Detection of Total and Glycated Hemoglobin in Human Whole Blood. Scientific Reports 7: 1016.

30. Zhu L, Liu Y, Yang PY, Liu BH (2015) Label-free Aptasensor based on Electrodeposition of Gold Nanoparticles on Graphene and Its Application in the Quantification of Adenosine Triphosphate. Electrochimica Acta 172: 88-93.

31. Lin ZY, Luo F, Liu QD, Chen LF, Quu B, et al. (2011) Signal-on electrochemiluminescent biosensor for ATP based on the recombination of aptamer chip. Chemical Communications 47: 8064-8066.

32. Chen H, Chen Q, Zhao YY, Zhang F, Yang F, et al. (2014) Electrochemiluminescence aptasensor for adenosine triphosphate detection using host-guest recognition between metallocyclodextrin complex and aptamer. Talanta 121: 229-233.

33. Liu BQ, Zhang B, Chen GN, Yang HG, Tang DP (2014) Metal sulfidefunctionalized DNA concatamer for ultrasensitive electronic monitoring of ATP using a programmable capillary-based aptasensor. Biosensors \& Bioelectronics 53: 390-398. 